http://dx.doi.org/10.32911/as.2017.v10.n2.170

Aporte Santiaguino. 10 (2), 2017: 283-294

ISSN 2070-836X

\title{
Tipificación y modelado gráfico de fincas agrícolas en la cuenca hidrográfica media y baja del río Supe (provincia de Barranca)
}

Characterization and graphic modeling of agricultural farm in the middle and lower water basin of the Supe river (province of Barranca)

Lorenzo Ayora Garagate, ${ }^{1}$ José Ramírez Maldonado ${ }^{1}$ y Luis Javier Cabana ${ }^{1}$

\section{RESUMEN}

El objetivo de la investigación fue clasificar y tipificar a los productores que conducen fincas agrícolas con cultivos prevalentes en la cuenca hidrográfica media y baja del río Supe; representar el desempeño actual de las fincas, mediante diagramas cualitativos de flujo; y diseñar mediante modelos de representación gráfica, los sistemas de fincas agrícolas mejorados; proponiendo alternativas técnicas de mejora para los niveles de agroecosistema, finca y región. La metodología utilizada consistió en efectuar el análisis multivariado utilizando un programa estadístico, seleccionando las variables más pertinentes, y determinando las tipologías de productores. Se identificaron limitaciones y potencialidades aplicando el análisis FODA, luego se procedió a jerarquizar los problemas clasificándolos según su grado de causalidad o consecuencia; se determinó cuáles son los problemas activos, pasivos, críticos e indiferentes; y su impacto en los sistemas agropecuarios de la zona en estudio. Para el diseño de las fincas, se construyeron modelos gráficos cualitativos a partir de las tipologías encontradas, considerando los datos económicos y técnicos que caracterizan los sistemas de finca.

Palabras clave: sustentabilidad; fincas; modelado gráfico; tipologías.

\begin{abstract}
The aim of the research was to classify and to typify leading producers farms with prevalent crops in the average and low hydrographic basin of the Supe river; to represent the current performance of farms, by qualitative flowcharts, and design models using graphical representation the improved systems of agricultural farms improved; proposing technical alternatives for improvement agroecosystem levels, farm and region. The used methodology consisted of effecting the multivariate analysis
\end{abstract}

1 Universidad Nacional Santiago Antúnez de Mayolo. Huaraz, Perú. 
using a statistical program, selecting the most relevant variables, and determining the typology of producers. Limitations and potentialities were identified applying the FODA analysis, then the problems were classified according to their degree of causality or consequence; it was determined which are the active, passive, critical and indifferent problems; and its impact on agricultural systems in the area under study. For the design of the farms, qualitative graphical models were constructed from the typologies found, considering the economic and technical data that characterize the farm systems.

Keywords: sustainability; farms; graphic modeling; typologies.

\section{INTRODUCCIÓN}

La investigación se orienta a encontrar los tipos de productores más representativos de la zona, elaborar modelos que grafiquen el desempeño de los sistemas, identificar factores limitantes y recursos potencialmente productivos en el área, y plantear alternativas técnicas que contribuyan a mejorar el desempeño de las fincas de la cuenca media y baja del río Supe.

Para identificar los tipos de productores que predominan en la región se efectúa el “análisis multivariado" utilizando el programa SPSS. Para ello se selecciona las variables más pertinentes de la base de datos; para los estudios de caso se selecciona un productor por cada tipología encontrada procurando que sea lo más representativo posible. Se recoge variables generales de la unidad de producción, así como variables sociales, económicas y técnicas que aplica cada productor. Para el diseño de fincas, primero se elabora modelos cualitativos de flujo a partir de la tipología encontrada, considerando los datos económicos y técnicos que caracterizan los sistemas de finca. Seguidamente se identifica limitaciones y potencialidades; se aplica el análisis tipo FODA, para ello se hicieron talleres participativos con los productores.

Los resultados que proporcione al final este trabajo de investigación, será de gran utilidad para diseñar estrategias y planes de desarrollo rural sostenible, y la práctica de una agricultura sustentable.

\section{MATERIALES Y MÉTODOS}

El ámbito de estudio comprende la parte media y baja de la cuenca hidrográfica del río Supe. Distrito de Supe; provincia de Barranca, departamento de Lima. La ubicación geográfica es:

$$
\begin{array}{ll}
\text { Latitud Sur } & : 10^{\circ} 50^{\prime} 29^{\prime \prime}-10^{\circ} 54^{\prime} 30^{\prime \prime} \\
\text { Longitud Oeste } & : 77^{\circ} 43^{\prime} 04^{\prime \prime}-77^{\circ} 27^{\prime} 50^{\prime \prime} \\
\text { Altitud } & : 0.0 \text { a } 1,250.0 \text { m.s.n.m. }
\end{array}
$$




\section{Materiales}

Software: SPSS V.21, Microsoft Office (Word y Excel), Auto-CAD. V 13

Materiales de escritorio: Ordenador de última generación, papeles bond diversos tamaños, Mapas y planos del IGN, base de datos de la Junta de Usuarios del Valle Supe, Impresora y Plotter.

Material de campo: GPS, cámara fotográfica y filmadora, encuestas y libretas de campo.

\section{Metodología}

Tipología de productores

Para identificar los tipos de productores que predominan en la zona se efectuó el "análisis multivariado" utilizando el programa estadístico SPSS V.21. Para ello se seleccionó las variables de mayor importancia e influencia de la base de datos. Para los estudios de caso se seleccionó un productor por cada tipología encontrada procurando que sea lo más representativo posible. Se recogió variables generales de la unidad de producción, variables sociales, económicas y técnicas que aplica cada productor (Escobar y Berdegue. 1990)

Diseño de fincas

a. Elaboración de modelos cualitativos. A partir de la tipología encontrada, se elaboraron modelos cualitativos de flujo, considerando los datos económicos y técnicos que caracterizan los sistemas de finca.

b. Identificación de limitaciones y potencialidades. Se aplicó el análisis tipo FODA, para ello se realizaron talleres participativos con los productores en la cuenca media y baja del río Supe. Se identifica las limitaciones y potencialidades que caracterizan la problemática de la zona. Enseguida se procedió a jerarquizar los problemas.

c. Jerarquización de problemas. Incluyó la construcción de un árbol de limitaciones, clasificando los problemas según su grado de causalidad o consecuencia y la aplicación de la "Matriz de Vester" para determinar cuáles son los problemas activos, pasivos, críticos e indiferentes.

d. Diseño de alternativas técnicas. Formulación de las ofertas tecnológicas con el fin de efectuar mejoras en los sistemas de producción existentes. 


\section{RESULTADOS}

\section{Tipología de productores y diseño de fincas}

Tipología de productores.- Se determinó la existencia de tres tipos de productores en la cuenca media y baja del río Supe; Tipo I: Pequeños agricultores con cultivos perennes extensivos y de economía restringida; Tipo II: Pequeños agricultores con actividad complementaria no agrícola y economía de autosubsidio; Tipo III: Medianos agricultores con cultivos perennes intensivos y economía de capitalización. La variable que distingue estos tres tipos es el "ingreso económico neto anual" que tiene la unidad de producción (finca). En la tabla 1 se observa las variables que fueron consideradas para elaborar las tipologías y se detalla las características por cada tipo encontrado.

Tabla 1. Características de los tipos de productores de la cuenca media y baja del río Supe

\begin{tabular}{|c|c|c|c|}
\hline Variable & Tipo I & Tipo II & Tipo III \\
\hline $\begin{array}{l}\text { Grado de escola- } \\
\text { ridad del jefe de } \\
\text { familia }\end{array}$ & $\begin{array}{l}\text { Analfabeto }(8.70 \% \\
\text { Educación primaria }(54.05 \%) \\
\text { Educación secundaria }(45.95 \%)\end{array}$ & $\begin{array}{l}\text { Analfabeto }(6.125) \\
\text { Educación primaria }(48.98 \%) \\
\text { Educación secundaria }(40.82 \%) \\
\text { Educación técnica }(4.08 \%)\end{array}$ & $\begin{array}{l}\text { Educación primaria }(25 \%) \\
\text { Educación secundaria }(66.67 \%) \\
\text { Educación técnica }(8.33 \%)\end{array}$ \\
\hline $\begin{array}{l}\text { Actividad econó- } \\
\text { mica principal }\end{array}$ & $\begin{array}{l}\text { Solo agricultura }(86.5 \%) \\
\text { Pequeños ganaderos }(2.7 \%) \\
\text { Comercio }(2.7 \%) \text {, peón }(8.1 \%)\end{array}$ & $\begin{array}{l}\text { Solo agricultura }(83.67 \%) \\
\text { Turismo }(4.08 \%) \\
\text { Comercio }(8.16 \%) \text {, otras }(4.09 \%)\end{array}$ & $\begin{array}{l}\text { Solo agricultura }(75 \%) \\
\text { Pequeños ganaderos }(6.0 \%) \\
\text { Comercio }(12.5 \%) \text {, otras }(6.5\end{array}$ \\
\hline $\begin{array}{l}\text { Propiedad de la } \\
\text { tierra }\end{array}$ & $\begin{array}{l}\text { Con titulo de propiedad } \\
(51.4 \%) \\
\text { Sin título de propiedad }(48.6 \%)\end{array}$ & $\begin{array}{l}\text { Con título de propiedad }(71.4 \%) \\
\text { Sin título de propiedad }(28.6 \%)\end{array}$ & $\begin{array}{l}\text { Con título de propiedad }(91.67 \%) \\
\text { Sin título de propiedad }(8.33 \%)\end{array}$ \\
\hline $\begin{array}{l}\text { Extensión de la } \\
\text { finca (ha) }\end{array}$ & Pequeña (1-2.0 ha) & Pequeña (2 - 4 ha) & Mediana (4 - 10 ha) \\
\hline $\begin{array}{l}\text { Cultivos prevalen- } \\
\text { tes }\left(^{*}\right)\end{array}$ & $\begin{array}{l}\text { Maíz amarillo }(21.6 \%) \text {, caña } \\
(5.4 \%) \\
\text { Maíz morado }(24.3 \%), \text { Ají } \\
\text { paprika }(2.7 \%) \\
\text { Palta }(16.2 \%) \text {,maracuyá }(5.4 \%) \\
\text { Otros }(24.4 \%)\end{array}$ & $\begin{array}{l}\text { Maíz amarillo }(36.7 \%) \text {, caña } \\
(20.40 \%) \text {, } \\
\text { Ají paprika }(6.1 \%) \\
\text { Palta }(10.2 \%) \text {,maracuyá }(4.1 \%) \\
\text { Otros }(22.5 \%)\end{array}$ & $\begin{array}{l}\text { Maíz amarillo }(25 \%) \text {, caña } \\
(16.7 \%) \text {, } \\
\text { Ají paprika }(16.7 \%) \\
\text { Palta }(8.3 \%), \text { maracuyá }(25 \%) \\
\text { Otros }(8.3 \%)\end{array}$ \\
\hline $\begin{array}{l}\text { Ingreso económi- } \\
\text { co neto anual }(* *)\end{array}$ & $\begin{array}{l}\text { Muy bajos }(43.24 \%),(6000- \\
8400 \mathrm{~S}) \\
\text { Bajos }(56.76 \%),(9000-9600 \mathrm{~S})\end{array}$ & $\begin{array}{l}\text { Ingresos bajos }(53.06 \%)(10200- \\
10800 \mathrm{~S}) \\
\text { Ingresos medios }(46.94 \%)(13200- \\
14400 \mathrm{~S})\end{array}$ & $\begin{array}{l}\text { Ingresos medios }(66.7 \%)(16800- \\
18000 \mathrm{~S}) \\
\text { Ingresos altos }(33.3 \%)(19200- \\
21600 \mathrm{~S})\end{array}$ \\
\hline Agua para riego & $\begin{array}{l}\text { No tiene riego }(24,3 \%) \\
\text { Sí tiene riego }(75,7 \%)\end{array}$ & $\begin{array}{l}\text { No tiene riego }(12.65 \%) \\
\text { Sí tiene riego }(87.35 \%)\end{array}$ & $\begin{array}{l}\text { No tiene riego }(8.33 \%) \\
\text { Sí tiene riego }(91.67 \%)\end{array}$ \\
\hline Crédito & $\begin{array}{l}\text { No tiene acceso al crédito } \\
(54,1 \%) \\
\text { Sí accede al crédito }(45,9 \%)\end{array}$ & $\begin{array}{l}\text { No tiene acceso al crédito } \\
(28.57 \%) \\
\text { Si accede al crédito }(71.43 \%)\end{array}$ & $\begin{array}{l}\text { No tiene acceso al crédito }(8.33 \%) \\
\text { Sí accede al crédito }(91.67 \%)\end{array}$ \\
\hline $\begin{array}{l}\text { Sistemas de } \\
\text { producción predo- } \\
\text { minantes }\end{array}$ & $\begin{array}{l}\text { Cultivos perennes para la venta } \\
(27 \%) \\
\text { Cultivos transitorios- para venta } \\
(41.27 \%) \\
\text { Pequeña ganad. y crianzas } \\
\text { menores }(27.5 \%) \\
\text { Otras actividades }(3.8 \%)\end{array}$ & $\begin{array}{l}\text { Cultivos perennes para la venta } \\
(18.2 \%) \\
\text { Cultivos transitorios- para venta } \\
(43.5 \%) \\
\text { Pequeña ganad. y crianzas meno- } \\
\text { res }(23.7 \%) \\
\text { Otras actividades }(14.6 \%)\end{array}$ & $\begin{array}{l}\text { Cultivos perennes para la venta } \\
(48.75 \%) \\
\text { Cultivos transitorios- para venta } \\
(31.35 \%) \\
\text { Pequeña ganad. y crianzas meno- } \\
\text { res }(17.4 \%) \\
\text { Otras actividades }(2.5 \%)\end{array}$ \\
\hline Capacitación & $\begin{array}{l}\text { Sí ha recibido capacitación } \\
(21.6 \%) \\
\text { No ha recibido capacitación } \\
(78.7 \%)\end{array}$ & $\begin{array}{l}\text { Sí ha recibido capacitación } \\
(40.82 \%) \\
\text { No ha recibido capacitación } \\
(59.18 \%)\end{array}$ & $\begin{array}{l}\text { Sí ha recibido capacitación }(75 \%) \\
\text { No ha recibido capacitación }(25 \%)\end{array}$ \\
\hline
\end{tabular}


Aplicando el software SPSS, se graficó el dendrograma (anexo 5) y se observó las tres tipologías existentes. Se distingue la estructura de agrupación por tipo, el número de agricultores que conforman y el grado de afinidad que hay entre ellos. Así se tiene que el Tipo I agrupa a 38 agricultores; el Tipo II, 46 agricultores y el Tipo III, 14 agricultores haciendo un total de 98 agricultores que conforman toda la población muestreada.

Identificación de limitaciones y potencialidades

Con la participación de grupos involucrados, líderes y productores de la zona se realizó el análisis FODA.

1. Identificación de problemas. Los que se detallan en la tabla 2.

2. Jerarquización de problemas. Se construye la matriz de Vester (Chaparro, 1995).

Tabla 2. Matriz de Vester para los problemas identificados en la CAU Caral- Supe-Lima

\begin{tabular}{|c|c|c|c|c|c|c|c|c|c|c|}
\hline & & & & & & & & & $\begin{array}{l}\text { TOTAL } \\
\text { ACTIVOS }\end{array}$ & \\
\hline PROBLEMAS & P1 & P2 & P3 & P4 & P5 & P6 & P7 & P8 & $(X)$ & I \\
\hline $\mathrm{P} 1=$ Bajo precio de los cultivos & 0 & 1 & 0 & 1 & 2 & 2 & 3 & 1 & 10 & $\mathrm{~N}$ \\
\hline P2 = Escaso apoyo del Estado & 1 & 0 & 2 & 3 & 1 & 2 & 1 & 2 & 12 & $F$ \\
\hline P3 = Corrupción de dirigentes y adm & 0 & 2 & 0 & 2 & 3 & 2 & 1 & 3 & 13 & $\mathrm{~L}$ \\
\hline P4 = Baja productividad & 1 & 2 & 2 & 0 & 3 & 2 & 2 & 1 & 13 & $\mathrm{U}$ \\
\hline P5 = Venta de activos de la CAO & 1 & 1 & 1 & 2 & 0 & 2 & 2 & 2 & 11 & $E$ \\
\hline P6 = Escasa innovacion tecnologica & 2 & 1 & 0 & 3 & 0 & 0 & 2 & 1 & 9 & $\mathrm{~N}$ \\
\hline P7 = Migración de la población juvenil & 0 & 0 & 1 & 1 & 1 & 3 & 0 & 1 & 7 & $\mathrm{C}$ \\
\hline P8 = Desorganización institucional & 0 & 1 & 2 & 2 & 2 & 3 & 3 & 0 & 13 & I \\
\hline \multirow[t]{2}{*}{ TOTAL PASIVOS (Y) } & 5 & 8 & 8 & 14 & 12 & 16 & 14 & 11 & & A \\
\hline & \multicolumn{4}{|c|}{ DEPENDENCIA } & & & & & & \\
\hline
\end{tabular}

En un plano cartesiano se determina la ubicación de cada problema a través de un punto en el eje de coordenadas $\mathrm{x}, \mathrm{y}$.

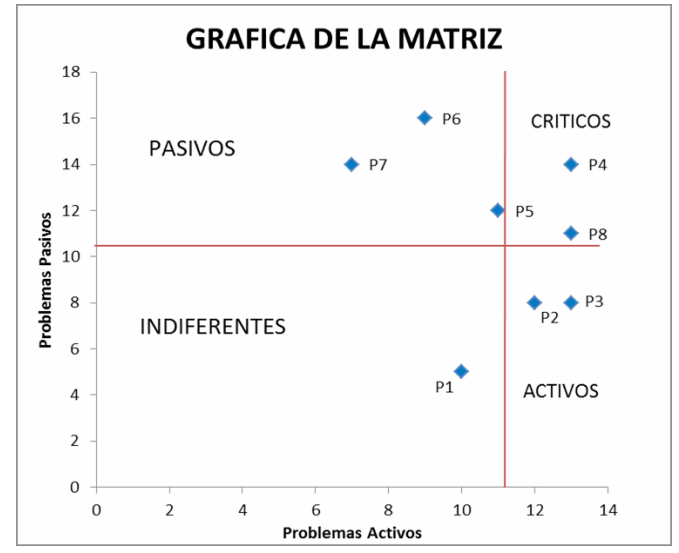

Figura 1. Calificación de problemas según su grado de causalidad en la CAU Caral 
La figura 1 muestra los problemas existentes y su calificación de acuerdo a los resultados obtenidos en la tabla 2.

3. Construcción del árbol de problemas. Con los resultados de la tabla 2 y de la figura 1 , se construyó el árbol de problemas que se presenta en el anexo 6.

\section{Diseño de fincas}

Para el diseño, se utilizó los resultados encontrados en etapas previas de evaluación y análisis. Se toma como referencia central las tres tipologías encontradas. Se construyeron modelos de representación gráfica para cada tipología encontrada (figuras 2, 3, 4, 5,6 y 7), estos son diagramas cualitativos que sintetizan y muestran el funcionamiento de las fincas con los sistemas de producción prevalentes.

Diseño actual. Se ha construido modelos de representación cualitativa de las fincas más representativas de la zona, mediante diagramas que muestran las características del subsistema socio económico (la familia campesina) y de los agroecosistemas (AGES) o campos de cultivo y núcleos de crianza. La figura 2 muestra el diseño para la tipología tipo I, de forma análoga se construye los modelos gráficos para las dos tipologías restantes (figuras 4 y 6). Las fincas tipo I producen maíz morado, palta, maracuyá, cultivos alimenticios y crianzas como porcinos, aves y cuyes. El agricultor vende parte de su cosecha los cultivos alimenticios (camote, zapallo, frejol, lechugas y otros) y las crianzas se destinan en un gran porcentaje para el autoconsumo; compra insumos y parte de comestibles del mercado; no accede al crédito y paga la mano de obra. Las fincas tipo II producen maíz amarillo, ají paprika, caña de azúcar y palta para la venta; así como cultivos alimenticios y crianzas menores para el autoconsumo y para la venta. El agricultor accede al crédito, alquila la mano de obra y maquinaria agrícola; adquiere bienes de producción. Las fincas tipo III tienen producción intensiva de maíz amarillo, caña de azúcar, ají paprika, así como cultivos alimenticios para el autoconsumo y producción de aves y cuyes para el mercado. El agricultor accede al crédito, alquila mano de obra, alquila tractores agrícolas. Es emprendedor y tiene capacidad de gestión, además capitaliza la finca mediante la compra de bienes de producción.

Diseño propuesto. Los sistemas propuestos se sustentan en la necesidad de optimizar los recursos, incluyendo nuevos cultivos y crianzas, ordenarlos de tal manera que puedan mejorar los rendimientos biológicos y económicos de la finca. Así para cada tipología de agricultor se construyeron modelos de fincas mejoradas (figuras 3, 5, 7); en ellas se observan las propuestas de corrección y mejora de los sistemas estudiados. La Figura 3 muestra el diseño propuesto para los agricultores tipo I, en donde se incluye los cultivos de lúcuma y pecanas, se propone el uso de abonos orgánicos, se incorpora la crianza de abejas para la producción de miel, polen y jalea para el mercado y la producción de porcinos, aves y cuyes para la venta. Además, se incorpora riego tecnificado para los frutales con la finalidad de brindarle mayor productividad y continuidad a la producción agrícola durante todo el año. Se proyecta incorporar el uso de máquinas agrícolas en mantenimiento de los cultivos. 


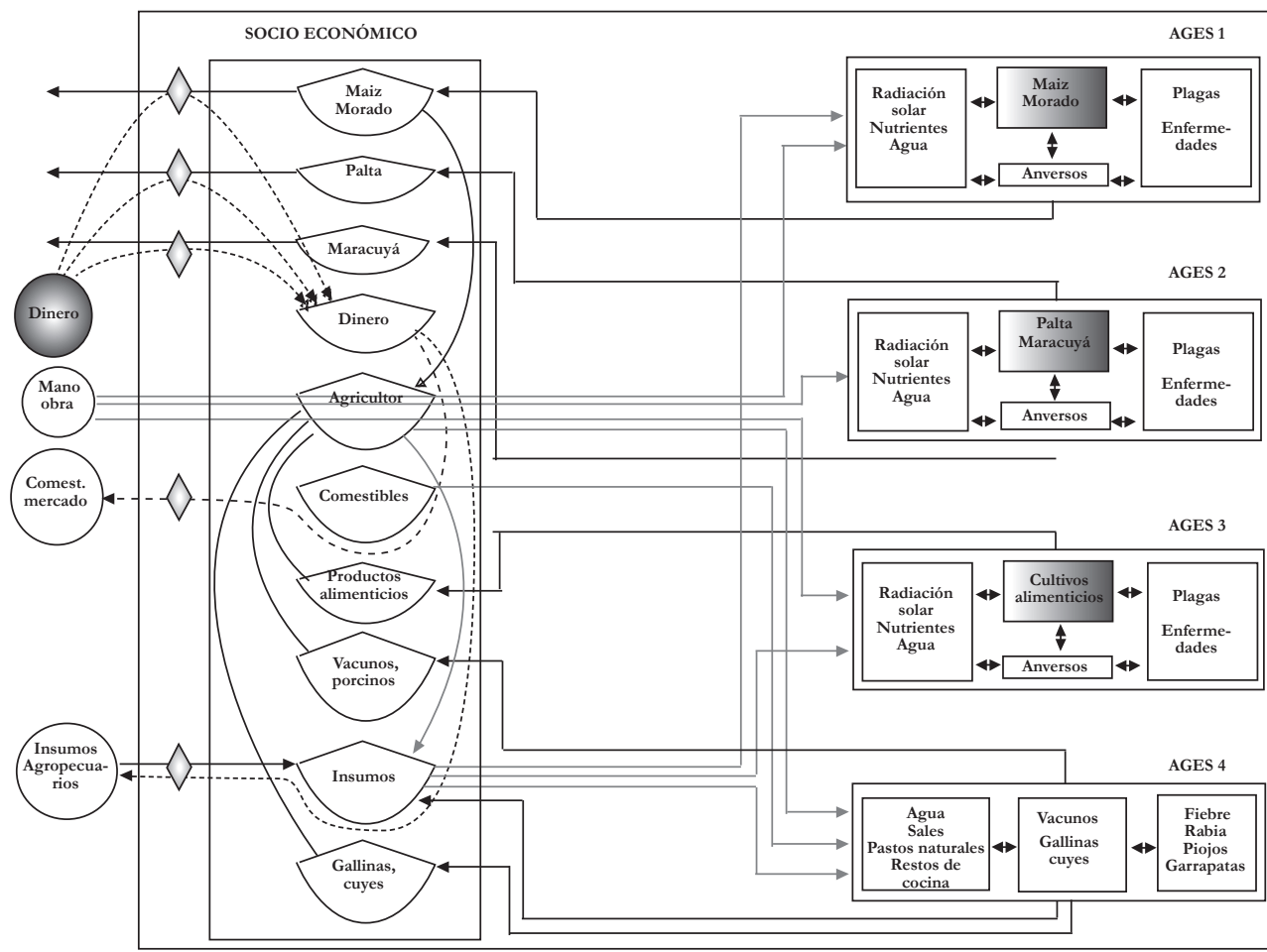

Figura 2. Diagrama cualitativo de flujos del sistema de finca de los agricultores Tipo I- Diseño actual

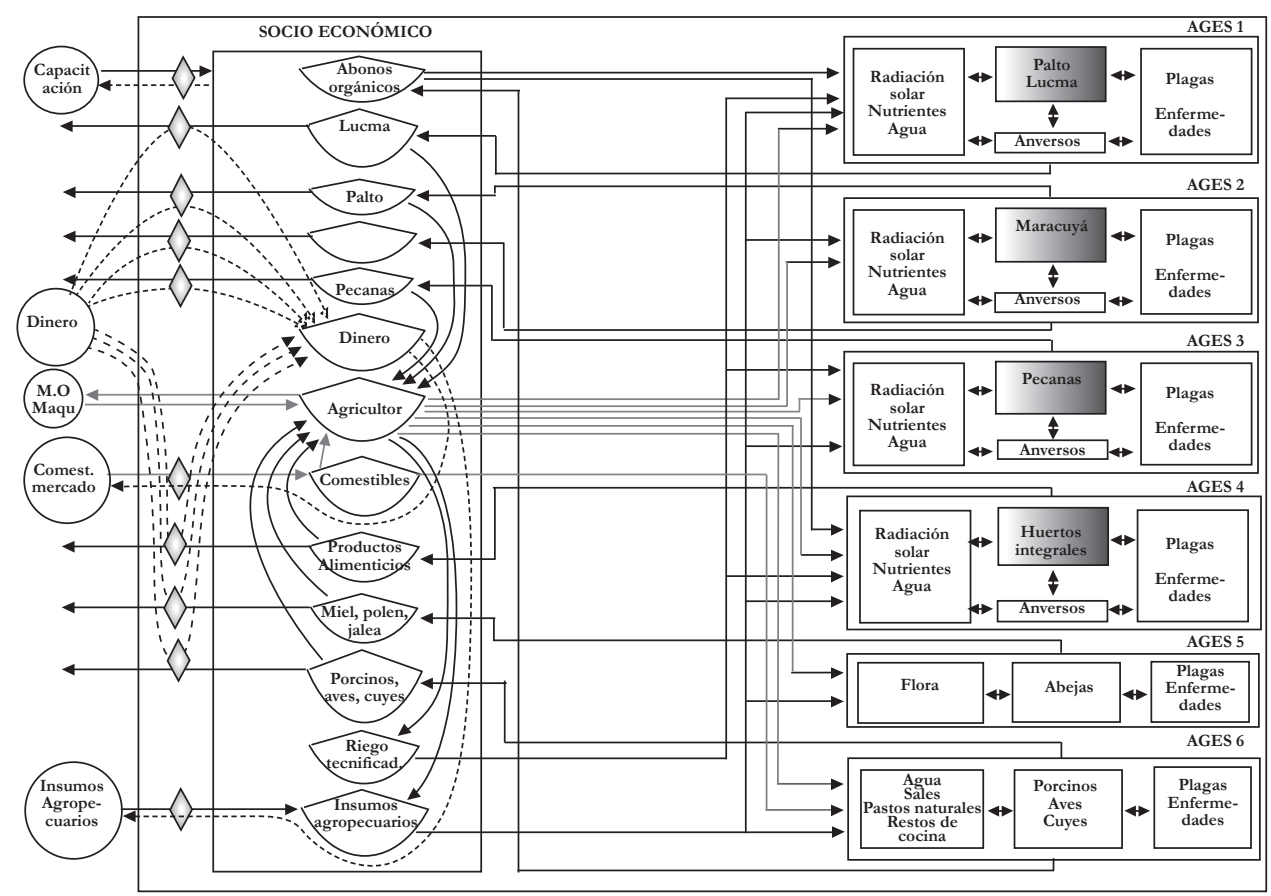

Figura 3. Diagrama cualitativo de flujos del sistema de finca de los agricultores tipo I- Diseño propuesto 


\section{Propuesta de alternativas tecnológicas}

Con el diagnóstico de la zona estudiada, la evaluación de sustentabilidad de fincas y la identificación de limitantes y potencialidades, se proponen alternativas tecnológicas (anexo 7) para la zona intervenida en la investigación. En la tabla 3 se detalla las mejoras propuestas para la cuenca media y baja del río Supe desagregados en programas y subprogramas.

Tabla 3: Propuesta de mejoras para la zona de estudio

PROMOVER LA GESTIÓN SOSTENIBLE DE LOS RECURSOS, ESPECIALMENTE AGUA, SUELO Y BOSQUES

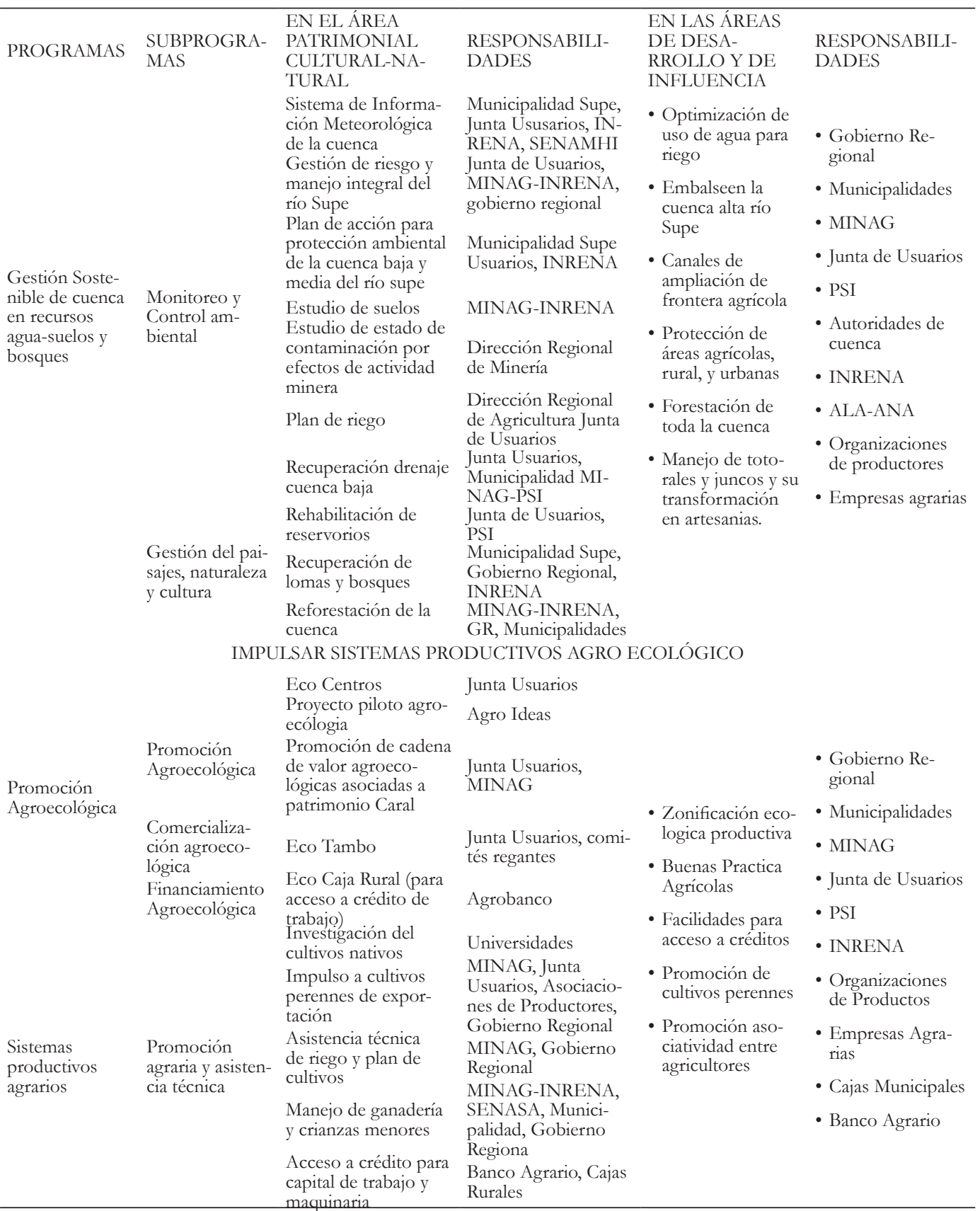




\section{DISCUSIÓN}

Los agricultores del Tipo I son considerados como de "economía restringida" porque el 43,24 \% de ellos tienen ingresos netos muy bajos durante un año (S/ 6000-8400) y el $56,76 \%$ tienen ingresos bajos (S/ 9000-9600); la economía precaria del que disponen solo les alcanza para subsistir. Para Merma y Julca (2012), los agricultores del tipo I se ubican entre el nivel mínimo con el cual una familia puede sobrevivir y el mínimo necesario para satisfacer las necesidades de la familia y reponer los medios de producción, lo que significa un estancamiento de los medios de producción y una condición económica precaria por la venta poco rentable de sus productos agropecuarios y la autosubsistencia. Los que se sitúan en el nivel inferior pueden llegar a abandonar la finca (migrar) o vender su predio.

Los agricultores del tipo II son considerados como de "economía de autosubsidio" porque el 53,06 \% tiene ingresos bajos ( $\mathrm{S} / 10$ 200-10 800) y el 46,94 \% tienen ingresos medios (S/ 13 200-14 400). El ingreso económico por actividades no agrícolas subsidia la economía agrícola y permite la reproducción de algunos medios de producción.

Los agricultores del tipo III son considerados como de "economía de capitalización" el 66,7\% obtienen ingresos medios (S/ 16 800-18 000) y el 33,3\% obtienen ingresos altos (S/ 19 200-21 600 Soles), estos tienen una economía suficiente para reproducir los medios de producción y capitalizar la finca.

Estudios sobre tipología de productores se han efectuado en nuestro país, y en varios países latinoamericanos, en función a variables priorizadas según su importancia relativa en la actividad agropecuaria, reportándose resultados similares al presente estudio. Así Merma, I; (2012) realizo la evaluación y diseño de fincas con cultivos prevalentes en la selva alta del Perú (Cusco).

Los resultados de las tipologías halladas permiten inferir que en la estructura socio-económica de las fincas de la zona estudiada coexisten dos lógicas de producción: de racionalidad capitalista y de racionalidad tradicional o de subsistencia. Los medios de producción (tierra, mano de obra, bienes de capital, y otros.) son utilizados por el agricultor en forma combinada para obtener una producción que satisfaga en primer lugar las necesidades básicas de la agricultura familiar y luego utiliza los excedentes para la venta. Cuando los medios de producción están en mínima cantidad, la agricultura se vuelve una actividad económica poco o nada rentable. Esta situación obliga a los miembros de la familia campesina a emplear su mano de obra por salario; el agricultor busca nuevas fuentes de ingreso económico en ocupaciones fuera de su finca, trabajando en actividades que le generen un ingreso económico adicional (caso tipo II). Los cultivos perennes y los frutales se cultivan principalmente para la venta (50\%) y en menor proporción para el consumo familiar. La venta de los cultivos comerciales 
se utiliza para comprar bienes que no pueden ser producidos en la finca. La pequeña ganadería y crianzas menores es una actividad importante en la economía de la zona (58,33 a 63,27 \%), el animal en pie representa un ahorro para el agricultor, pues este puede vender el ganado en caso de presentarse alguna urgencia económica.

Las crianzas menores como el de gallinas, patos, pavos y cuyes son actividades generalizadas en casi todas las fincas; esta producción es principalmente para el autoconsumo familiar y venta.

\section{Diseño propuesto}

La propuesta de nuevos sistemas se basa en la necesidad de revisar experiencias (técnicas, económicas) de los cultivos. Los frutales y semillas comestibles tienen proyecciones positivas en el mediano plazo dependiendo del fortalecimiento de la economía mundial. Los productores que adopten más tecnología para mejorar el cultivo y su procesamiento, se beneficiarán; mientras que los no adoptadores seguirán en la crisis de bajos precios o abandonarán sus plantaciones (Toledo, 1994).

La fruticultura ofrece considerables beneficios sociales ya que las plantaciones pueden generar oportunidades de trabajo mejor remuneradas por tratarse de rubros que permiten encadenamientos, desde la fabricación de insumos para la producción, hasta la comercialización. La agroindustria rural es otro sector que está creciendo dando alternativas para el aprovechamiento de las frutas por grupos de pequeños empresarios, especialmente mujeres organizadas, quienes obtienen ingresos de las actividades de procesamiento de las frutas (IICA, 2008). Cultivos perennes (como caña de azúcar, maracuyá y espárragos), y árboles frutales constituyen excelentes componentes para diseñar sistemas agrícolas.

En la tipología tipo I, se aprecia el desempeño dinámico del subsistema socioeconómico, el hogar campesino y su administración de recursos; y los agroecosistemas de cultivos y crianzas con sus respectivos flujos e interacciones. Sus ingresos son bajos y cuando no vende sus productos se adapta a condiciones de autosubsistencia.

\section{Propuestas tecnológicas}

Las alternativas técnicas propuestas, como son las alternativas tecnológicas agropecuarias, la gestión sostenible de los recursos y los sistemas productivos agroecológicos; y su correcta aplicación por los productores, instituciones y agentes del desarrollo, contribuirán a mejorar el desempeño de los sistemas agropecuarios de la zona, optimizar el manejo de los recursos del medio y mejorar la calidad de vida de la población rural. 


\section{CONCLUSIONES}

Tipología de fincas

- Se encontró tres tipos de productores según la actividad económica principal (tabla 1) y el ingreso económico anual de la familia: Tipo I: Pequeños agricultores con cultivos perennes extensivos y de economía restringida; Tipo II: Pequeños agricultores con actividad complementaria no agrícola y economía de autosubsidio; Tipo III: Medianos agricultores con cultivos intensivos y economía de capitalización.

- Se encontró formas agrarias empresariales con prácticas de producción intensificadas, con acceso a ciertos servicios como riego, créditos y con unidades de producción de mediano nivel de productividad y con economía de capitalización; y las formas campesinas, caracterizadas por prácticas de producción no intensificadas con unidades de producción con bajos niveles de productividad y de economía restringida.

- Se elaboraron diseños gráficos que muestran el estado actual de las fincas y los diseños alternativos o mejorados para cada tipo de finca (I, II, III), en ellos se observan las propuestas de corrección y mejora de los sistemas estudiados.

Limitaciones y potencialidades

- Los principales factores externos que limitan el desarrollo agrario en la región son entre otros: los bajos precios de los productos, falta de infraestructura de riego, deficientes vías de comunicación, falta de mercados y falta de crédito agrario.

\section{Alternativas tecnológicas}

- Se identificaron alternativas tecnológicas para los productores, instituciones y agentes del desarrollo en la cuenca baja y media del río Supe; las mismas contribuirán a mejorar el desempeño de los sistemas agropecuarios, optimizar el manejo de los recursos y mejorar la calidad de vida de la población rural.

\section{REFERENCIAS BIBLIOGRÁFICAS}

Chaparro Anaya, Óscar. 1995. Análisis y priorización de problemas. En: Manual para la gestión de proyectos de desarrollo tecnológico. CORPOICA, Santa Fe, Bogotá.

Escobar, German y Berdegué, Julio. 1990. Tipificación de sistemas de producción agrícola. RIMISP. Santiago de Chile. Chile. Grafica Andes. 
IICA. (Instituto Interamericano de Cooperación para la Agricultura). 2008. La Fruticultura en Panamá: su potencial socioeconómico e iniciativas para su desarrollo. Costa Rica.

Merma, Isaías. 2012. Evaluación y diseño de fincas en selva alta bajo sistemas de cultivos prevalentes, en la Convención- Cusco. Tesis Ph.D. Lima. UNALM. Perú.

Toledo, Jorge. 1994. El desarrollo sostenible amazónico en una economía de mercado: un análisis crítico. En: Biodiversidad y Desarrollo Sostenible de la Amazonía en una Economía de Mercado. Seminario-Taller, Ucayali 11-15 octubre de 1994.

Recibido: 29/08/17

Aceptado: 31/10/17

\section{Correspondencia}

Lorenzo Ayora Garagate

lorenzoayora@hotmail.com 